\title{
Radiation-induced Grafting of Styrene onto Polyethylene Films for Preparation of Cation Exchange Membranes: Effect of Crosslinking ${ }^{+}$
}

\author{
M. M. Nasef ${ }^{1 *}$, H. Saidi ${ }^{2} \&$ A. H. Yahaya ${ }^{3}$ \\ ${ }^{1 \& 2}$ Business and Advanced Technology Centre, Universiti Teknologi Malaysia \\ Jalan Semarak, 54100 Kuala Lumpur, Malaysia \\ ${ }^{3}$ Department of Chemistry, Faculty of Science, Universiti Malaya \\ 50603 Kuala Lumpur, Malaysia
}

\begin{abstract}
Crosslinked cation exchange membranes bearing sulfonic acid groups (PE-g-PSSA/DVB) were prepared by radiationinduced grafting of styrene/divinylbenzene (DVB) mixtures onto low density polyethylene (PE) films followed by sulfonation reactions. The effect of addition of DVB ( 2 and 4\%) on the grafting behavior and the physico-chemical properties of the membranes such as ion exchange capacity, swelling and ionic conductivity were evaluated in correlation with grafting yield (Y\%). The structural and thermal properties of the membranes were also studied using differential scanning calorimetry (DSC) and thermal gravimetric analysis (TGA), respectively. Crosslinking with DVB was found to considerably affect the properties of the membranes in a way that reduces the swelling properties and enhances the chemical stability. The ion conductivity of the crosslinked membranes recorded a level of $10^{-2} \mathrm{~S} / \mathrm{cm}$ at sufficient grafting yield (28\%) despite the reduction caused by the formation of crosslinking structure. The results of this work suggest that membranes prepared in this study are potential alternatives for various electrochemical applications.
\end{abstract}

Keywords : Crosslinked cation exchange membranes, styrene, DVB, low density PE, radiation-induced grafting

\subsection{INTRODUCTION}

For many years, ion exchange membranes have attracted an increasing attention in a wide number of electrochemically driven membrane processes such as desalination of brackish water, concentration of salts, heavy metal removal for industrial waste water and recovery of organic acids [1,2]. Most of commercially available ion exchange membranes composed of copolymer of styrene and divinylbenzene (DVB) which are subsequently modified by sulfonation reaction. The selection of styrene/DVB copolymer has been particularly preferred because of its good workability and mechanical strength [3].

* Correspondence: M. M. Nasef (Tel: +603-26914020, Fax: +603-26911927, Email: mahmoudeithar@utmkl.utm.my)

Partially presented at Macromolecular Science and its Impact on Industries. 27-29 September, 2004. Petaling Jaya, Selangor, Malaysia 
Preparation of ion exchange membranes is often carried out by copolymerization using various routes such as bulk polymerization [4], latex [5], polymer blending [6] and inter-polymerization [7]. Commercial ion exchange membranes are also obtained by coating the ionic polymer onto a fabric or reinforcing materials [8]. However, the presence of the reinforcing material affects the transport properties and increases the resistance of these membranes. To improve the membrane preparation, photo-initiated copolymerization and radiation-induced copolymerization were considered [9]. However, the later method has been favored because it enables the formation of thinner defect free membranes with surface as well as bulk modification and simplifies the whole preparation process [1].

A number of studies have reported the preparation of strongly acidic cation exchange membranes by radiation-induced grafting of styrene (or it derivatives) onto polyethylene (PE) followed by sulfonation reactions [10-19]. The use of $\mathrm{PE}$ as polymer matrix was prompted by its high radiation resistance, excellent mechanical properties and cheapness. While most of the studies focused on improving the membranes transport and stability properties, very little efforts were spared to investigate the effect of crosslinking on the grafting behavior and on the various physico-chemical properties of these membranes as indicated by the scarce number of reports in literature [1].

In our previous study, we have reported the preparation of ion exchange membranes by the simultaneous radiation grafting of styrene into low density PE films and subsequent sulfonation [16]. The correlations between the grafting yield and the physico-chemical properties of membranes were established [17]. The thermal and structural properties of the membranes were also investigated [20]. The objective of the present study is to investigate the effect of crosslinking with DVB on the various properties (physico-chemical, thermal, structural and chemical stability) of the crosslinked cation exchange membranes referred as PE-g-PSSA/DVB. Non-crosslinked membranes denoted as PE-g-PSSA were used as references with respect to various grafting yields.

\subsection{EXPERIMENTAL}

\subsection{Materials}

PE films with thickness of $60 \mu \mathrm{m}$ were supplied by Al-Nasr Co. (Egypt). Styrene (>99\% purity) and DVB (65\% in ethylvinylbenzene) were purchased from Fluka and used without further purification. All solvents and chlorosulfonic acid (Fluka) were research grades and used as received. The PE films were purified with acetone and dried at $50^{\circ} \mathrm{C}$ under vacuum for 2 hours prior to grafting reaction.

\subsection{Membrane Preparation}

LDPE film of known size and weight was placed in a glass ampoule containing styrene monomer (50 vol\%) diluted in benzene. DVB was added to the grafting mixtures at the desired concentration. No other additives were added to the grafting mixture. The air was removed from the grafting mixture by flushing the ampoule with purified $\mathrm{N}_{2}$ for 10 minutes. The ampoule was then sealed and irradiated using $\gamma$-rays form a ${ }^{60} \mathrm{Co}$ source of dose rate of $1.32 \mathrm{kGy} / \mathrm{h}$ to a prescribed time. After completion of the grafting, the grafted film denoted as PE-g-PS was extracted, washed with toluene and soaked therein overnight to remove the excess monomer and homopolymer from the film surfaces. Details of the effect of various grafting parameters for grafting of styrene onto PE on the grafting yield and the properties of the obtained graft copolymer films were reported elsewhere [16]. The grafting yield 
(Y\%) was gravimetrically determined as the percentage of weight increase in PE film after grafting reaction using Equation (1):

$$
Y(\%)=\left[\left(W_{g}-W_{0}\right) / W_{0}\right] \times 100
$$

where, $W_{g}$ and $W_{0}$ are the weights of grafted and original PE films, respectively.

The grafted PE films were sulfonated in a mixture composes of 10 parts chlorosulfonic acid in 90 parts of 1, 1, 2, 2-tetrachloroethane $(v / v)$ for 2 hours at room temperature using the method described in a previous communication [17]. The sulfonated membranes were washed with deionized water a few times then hydrolyzed with $0.5 \mathrm{M} \mathrm{KOH}$ solution overnight and regenerated into the acid form by boiling with $1 \mathrm{M} \mathrm{HCl}$ for 2 hours. The samples were then washed free of excess $\mathrm{HCl}$ with deionized water a few times.

\subsection{Physico-chemical Properties}

The chemical composition of crosslinked and non-crosslinked membranes was studied by FTIR spectrometer (Perkin Elmer, Spectrum 2000 Explorer) equipped ATR having ZnS crystal fixed and $45^{\circ}$ angle, at ambient conditions in transmittance mode at wave number in the range of 500-4000 $\mathrm{cm}^{-1}$. Original PE and PE-g-PS films were used as references.

Ion exchange capacity (IEC) of the membranes was determined by back titration. Membrane samples in acid form were immersed into $0.5 \mathrm{M} \mathrm{KCl}$ solution overnight at room temperature. The protons $\left(\mathrm{H}^{+}\right)$released in the solution were titrated against standardized $0.05 \mathrm{M} \mathrm{KOH}$ solution by an automatic titrator (Metrohom, Switzerland) until pH 7 was reached. IEC per unit mass of the dry membrane $(\mathrm{mmol} / \mathrm{g})$ was calculated from the volume $\mathrm{KOH}$ solution consumed in titration after taking the weight of the dry membrane into account. The theoretical ion exchange capacity was calculated based on the assumption that the ratio of sulfonic acid groups to aromatic rings equal to unity.

The swelling of the membranes was determined by calculating the water uptake and the number of water molecules per sulfonic acid group $\left(\mathrm{nH}_{2} \mathrm{O} / \mathrm{SO}_{3}{ }^{-}\right)$. Dry membrane samples in the acid form were immersed in boiling deionized water until swelling equilibrium was achieved. The membrane samples were removed, the excess of water adhering to the surface was quickly blotted by absorbent papers and then the samples were weighed. The membrane water uptake was calculated using Equation (2):

$$
W(\%)=\left[\left(W_{w}-W_{d}\right) / W_{d}\right] \times 100
$$

where, $W_{w}$ and $W_{d}$ are the weights of wet and dried membranes, respectively.

Ionic conductivity of the membranes in the acid form was measured at room temperature by AC impedance spectroscopy. Measurements were performed using a frequency response analyzer (Solartron, 1250) in combination with an electrochemical interface (EG\&G Princeton Applied Research) at 0.01-100 kHz frequency range. Water swollen-membrane samples of circular shape were clamped between two stainless steel electrodes having disc-ends of $20 \mathrm{~mm}$ diameter and hosted in a home-made Teflon cell. The ionic conductivity was calculated by taking the measured resistance of the membranes and their thicknesses into consideration as described in a previous study [17].

The chemical stability of the membranes was tested by soaking in solutions of $3 \% \mathrm{H}_{2} \mathrm{O}_{2}$ containing $4 \mathrm{ppm} \mathrm{Fe}^{2+}$ at $70^{\circ} \mathrm{C}$ for 5 hours. The membranes were then dried and the losses in terms of weight and IEC were determined. 


\subsection{Melting Behavior and Crystallinity}

The melting behavior and the degree of crystallinity of the membranes were studied in correlation with the corresponding parameters in the original and grafted PE films using DSC analysis. Measurements were carried out using a Perkin-Elmer DSC-Pyris 1. Samples weighing 3-4 mg of sample was used, with the thermograms recorded under nitrogen flow $\left(10-5 \mathrm{~m}^{3} / \mathrm{min}\right)$ at a heating rate of $20^{\circ} \mathrm{C} / \mathrm{min}$ in temperature range of $25-150^{\circ} \mathrm{C}$. The melting temperature $\left(T_{\mathrm{m}}\right)$ and the heat of melting or enthalpy $\left(\Delta H_{\mathrm{m}}\right)$ were calculated from thermograms using the peak area under the melting peaks.

The degree of crytallinity $(\chi)$ of the original PE film was calculated using Equation (2):

$$
\chi(\%)=\left(\Delta H_{\mathrm{m}} / \Delta H_{\mathrm{m}}^{\circ}\right) \times 100
$$

where, $\Delta H_{\mathrm{m}}$ is the heat of melting of PE film and $\Delta H^{\circ}{ }_{\mathrm{m}}$ is the equilibrium heat of melting of $100 \%$ crystalline PE polymer, which equals to $290 \mathrm{~J} / \mathrm{g}$ [21].

Since the incorporated polystyrene has an amorphous nature, the crystallinity in the grafted PE films (PE-g-PS) and in the final sulfonated membranes (PE- $g$-PSSA) is always referred to the fraction of PE matrix.

The $\chi$ of PE-g-PS films is calculated using Equation (2) after correcting the recorded $\Delta H_{\mathrm{m}}$ by dividing over the weight fraction of PE in the grafted film $\left[\mathrm{W}_{P E(P E-\mathrm{g}-P S)}\right]$ obtained by:

$$
W_{P E(P E-\mathrm{g}-P S)}=\mathrm{m}_{P E} /\left(\mathrm{m}_{P E}+\mathrm{m}_{P S}\right)
$$

where, $\mathrm{m}_{P E}$ and $\mathrm{m}_{P S}$ are the weight fractions of $\mathrm{PE}$ and grafted polystyrene, respectively.

Similarly, $\chi$ of PE- $g$-PSSA or PE- $g$-PSSA/DVB membranes is calculated using Equation (2) after taking into account the weight fraction of PE in the membranes $\left[\mathrm{W}_{P E(P E-g-P S S A)}\right]$ calculated using Equation (4):

$$
W_{P E(P E-\mathrm{g}-P S S A)}=\mathrm{m}_{P E} /\left(\mathrm{m}_{P E}+\mathrm{m}_{P S S A}\right)
$$

where, $\mathrm{m}_{\mathrm{PSSA}}$ is the weight of sulfonated polystyrene grafts, which equals $\mathrm{m}_{\mathrm{PS}}+\mathrm{m}_{\mathrm{SO}_{3}}{ }^{-}+\mathrm{m}_{\mathrm{H}_{2} \mathrm{O}}$.

\subsection{Thermal Stability}

The thermal stability of the membranes were determined using TGA. Membrane samples of weight range of 5-10 mg were heated from room temperature to $600^{\circ} \mathrm{C}$ under nitrogen at a heating rate of $20^{\circ} \mathrm{C} / \mathrm{min}$ with TG7 (Perkin Elmer).

\subsection{RESULTS AND DISCUSSION}

\subsection{Effect of Addition of DVB on the Grafting Kinetics}

Crosslinked polystyrene sulfonic acid membranes (PE-g-PSSA/DVB) having various degrees of grafting were prepared by grafting styrene monomer solutions having 2 and 4\% DVB, respectively. The concentration of DVB is kept below 5\% (2-4\%) to allow the grafting reaction to proceed and minimize the gelation effect, which affects the handling of the grafted samples. The respective noncrosslinked membranes (PE-g-PSSA) were also prepared under similar grafting conditions and used 
for comparison purposes. The degree of grafting in each types of the membranes was controlled by varying the irradiation time while keeping styrene concentration at $50 \mathrm{vol} \%$ in benzene as a diluent and the dose rate at $1.32 \mathrm{kGy} / \mathrm{h}$ and under $\mathrm{N}_{2}$ atmosphere.

The relationships between the grafting yield (Y\%) and the irradiation time for grafting of styrene containing various concentrations of DVB onto PE films are shown in Figure 1. The grafting yield was found to increase linearly with the increase in the irradiation time regardless the DVB content in the bulk grafting mixture. This trend can be ascribed to the fact that the increase in the irradiation time leads to the availability of more free radicals in the grafting system, which enhances the grafting reactions leading to an increase in Y\%. The addition of DVB was found to increase Y\% and such increase is a function of DVB concentration in the bulk solution. For instance, the rate of grafting was found to increase from $1.25 \% / \mathrm{h}$ in the absence of DVB (0\%) to 1.69 and $2.44 \% / \mathrm{h}$ in the presence of 2 and $4 \%$ of DVB, respectively. This behavior can be attributed to the mutual reactivity of styrene and DVB, both of which compete to react with the free radicals formed in PE films with the grafting apparently taking place at a rate faster than crosslinking. If the crosslinking rate was faster than the rate of grafting, the graft growing chains would have been early terminated by DVB leading to a reduction in $\mathrm{Y} \%$. The higher grafting rates in the present grafting system (PE/Styrene/DVB) may be attributed to two mutual effects: 1) the scavenging of some of the formed radicals by the impurities (inhibitor) available in DVB and 2) the improvement in the swelling in grafting zone provided by PE matrix during the polymerization reaction. The former reduced the availability of DVB to the grafting zone whereas the latter allows more styrene in grafting zone and therefore, grafting is enhanced.

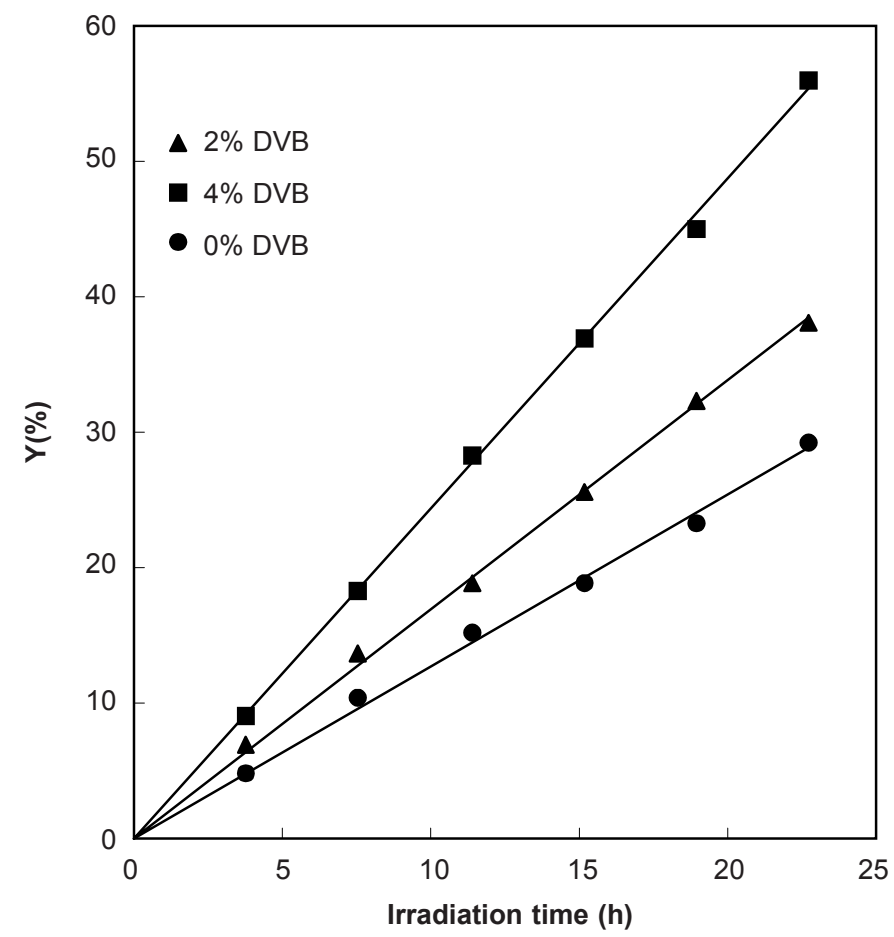

Figure 1 The relationship between grafting yield and irradiation time at various DVB contents. Grafting conditions are: styrene concentration, $50 \mathrm{vol} \%$; dose rate, $1.32 \mathrm{kGy} / \mathrm{h}$; diluent, benzene; temperature, $30^{\circ} \mathrm{C}$ and atmosphere, $\mathrm{N}_{2}$ 
These results are in a complete agreement with those reported for grafting of styrene (diluted with benzene) containing DVB below 5\% onto ethylene/tetrafluoroethylene (ETFE) compolymer films using pre-irradiation method [23]. Such superimposed behavior is mostly due to the similarity in the chemical structure of PE and ETFE, both of which possess $\left(-\mathrm{CH}_{2}-\mathrm{CH}_{2}-\right)$ basic units in their molecular structure. On contrary, grafting of styrene in presence of DVB onto fully fluorinated polymers such as poly(tetrafluoroethylene-co-hexafluoropropylene) (FEP) and poly (tetrafluoroethylene-coperfluorovinylether) (PFA) films was reported to reduce Y\% [23-24]. Such discrepancy may be attributed to the differences in the chemical structures of FEP and PFA, which have $\left(-\mathrm{CF}_{2}-\mathrm{CF}_{2}-\right)$ basic units and their poor swelling in the grafting solution, which prompt crosslinking at early stage of the reaction. Such early crosslinking competes with grafting causing deactivation in the polystyrene growing chains and hindering their mobility. Consequently, a decrease in the amount of monomer diffusing to the grafting sites takes place leading to a reduction in the grafting yield took place [2324].

\subsection{Verification of the Chemical Composition}

FTIR spectra of PE-g-PSSA and PE-g-PSSA/DVB membranes and their respective grafted and original PE films are shown in Figure 2. In the grafted film, the absorption band assigned to the aromatic rings of polystyrene was observed at 3050,1600 and $1494 \mathrm{~cm}^{-1}$, respectively. The couple of small bands at 698 and $750 \mathrm{~cm}^{-1}$ were assigned for the aromatic $\mathrm{CH}$ deformation of mono-substituted

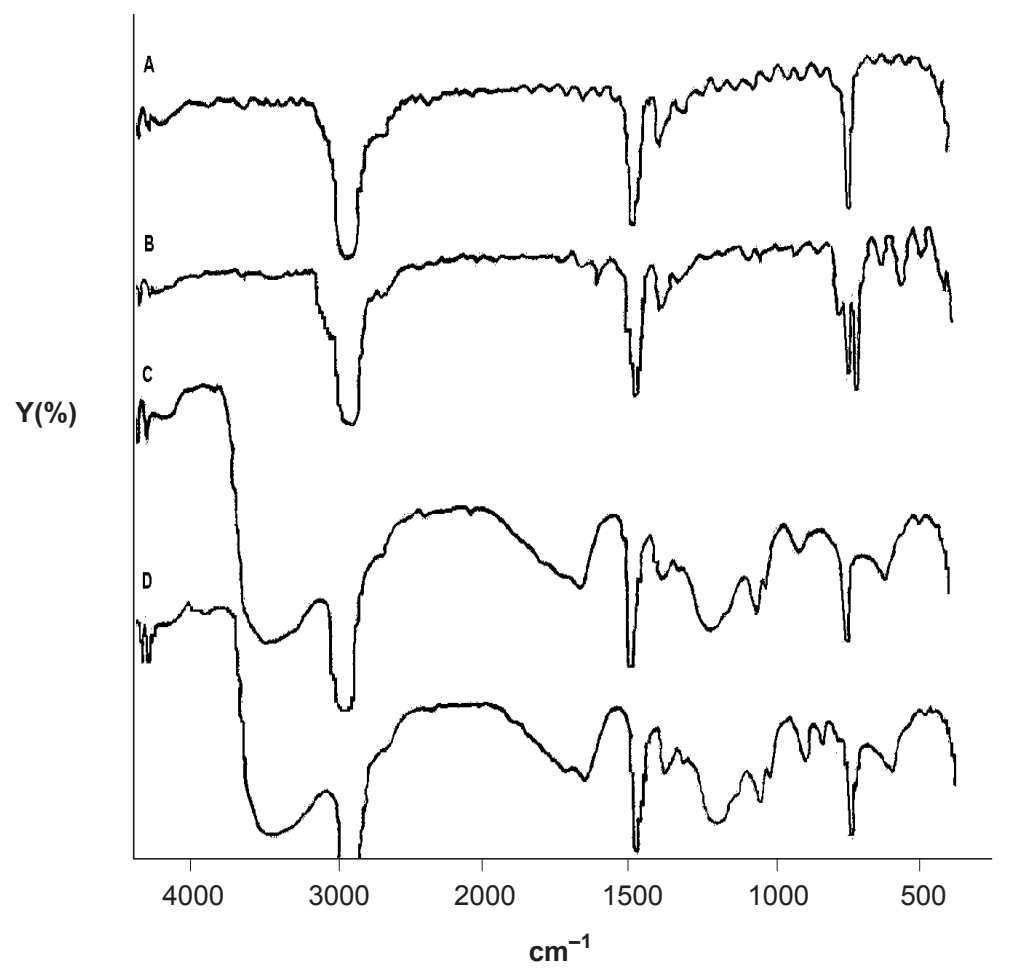

Figure 2 FTIR spectra of: (A) original PE film, (B) PE-g-PS (Y = 38\%), (C) PE-g-PSSA membrane $(\mathrm{Y}=38 \%)$ and $(\mathrm{D})$ PE-g-PSSA/DVB membrane $(\mathrm{Y}=38 \%)$ 
benzene ring of grafted polystyrene. These features confirm the grafting of styrene onto PE films. In PE-g-PSSA and PE-g-PSSA/DVB membranes, the absorption bands assigned to sulfonic acid groups were respectively, observed at 1123 and $1003 \mathrm{~cm}^{-1}$ while the spectra for $\mathrm{S}=\mathrm{O}$ stretching vibration were detected at $1300-1160 \mathrm{~cm}^{-1}$. The bands at 810 and $820 \mathrm{~cm}^{-1}$ are due to the di-substituted benzene ring of DVB crosslinks. The broad band at $3200-3600 \mathrm{~cm}^{-1}$ is due to the $\mathrm{OH}$ groups of water molecules strongly bound to $\mathrm{SO}_{3}{ }^{-}$groups by hydrogen bonding. The absorption bands of aromatic groups were obscured due to the strong intensity of the sulfonic groups and their associated hydration spheres. These results confirm the successful crosslinking of polystyrene during grafting reaction and sulfonation of crosslinked polystyrene grafts. Accordingly, a tentative molecular structure of crosslinked PE-g-PSSA/DVB membrane can be proposed as shown in Figure 3.

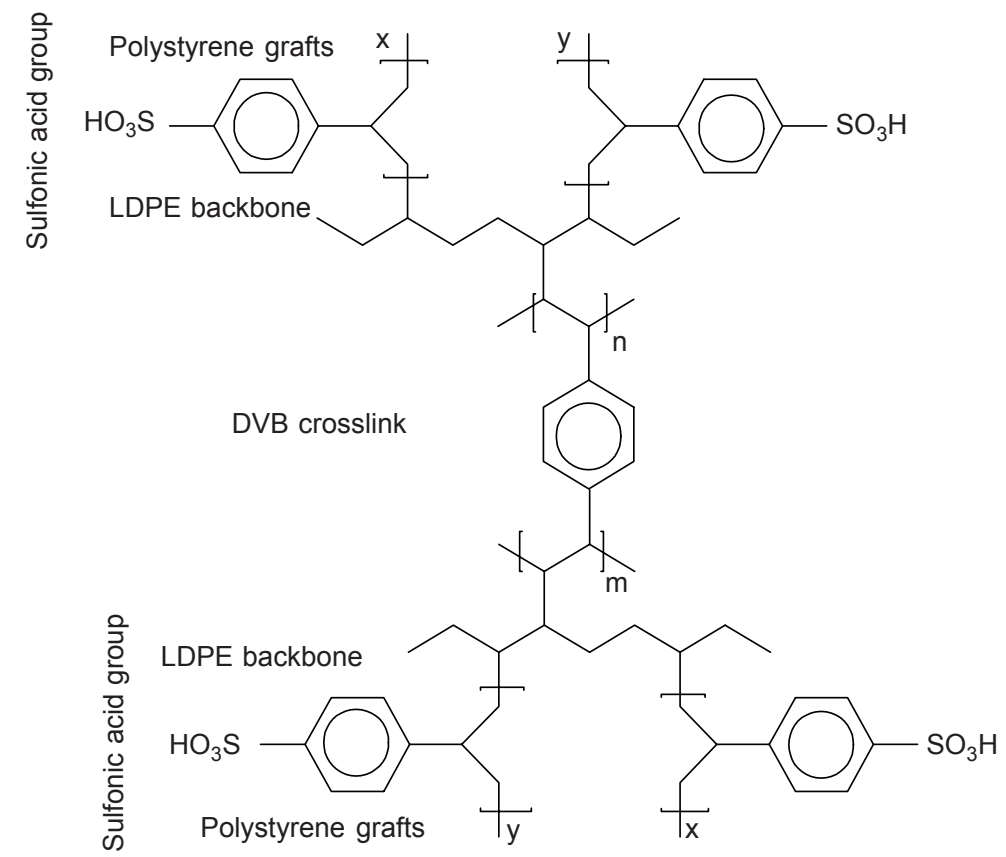

Figure 3 A tentative molecular structure of crosslinked PE-g-PSSA/ DVB membranes

\subsection{Physico-chemical Properties of the Crosslinked Membranes}

\subsubsection{Ion Exchange Capacity}

Figure 4 shows the relationship between the ion exchange capacity (IEC) and the grafting yield (Y\%) in PE-g-PSSA membranes at various DVB contents. The IEC of all membranes was found to increase gradually with the increase in Y\% regardless of crosslinking. The progressive increase in IEC of the membranes with the increase in Y\% can be understood based on the fact that the increase in the number of the incorporated polystyrene grafts gives rise to the number of the hosted sulfonic acid groups when subjected to sulfonation. On the other hand, the experimental values of IEC are in a good agreement with the theoretical ones (data is not shown in Figure 4). Particularly, for three 


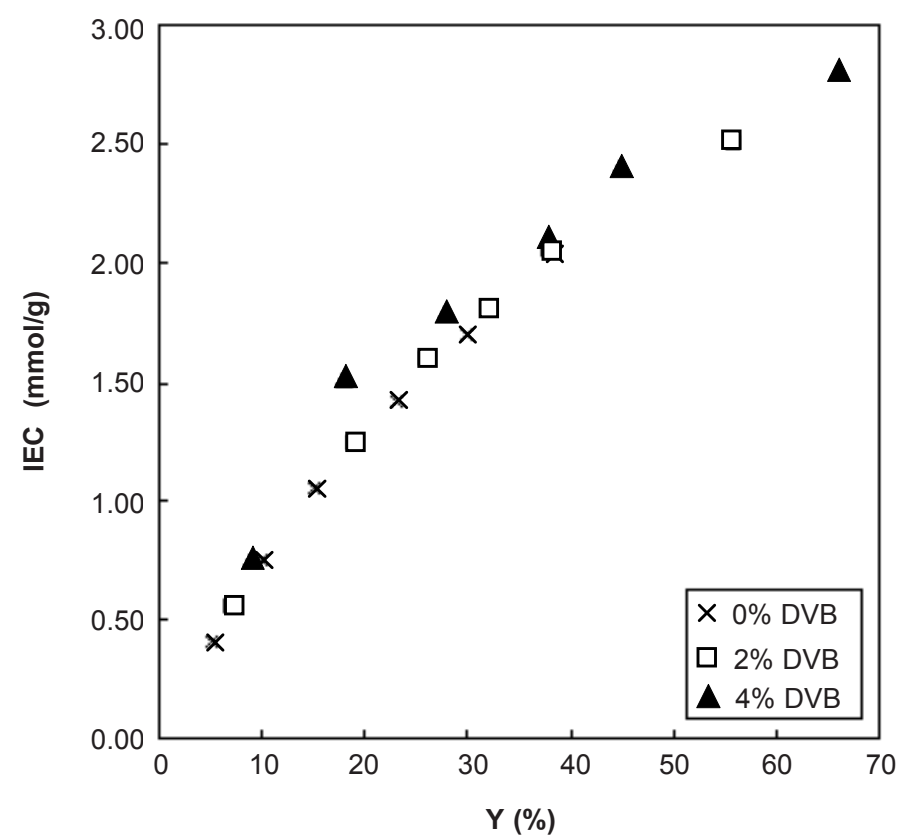

Figure 4 The relationship between ion exchange capacity and grafting yield for PE-g-PSSA membranes having various DVB contents

membranes having the same $\mathrm{Y} \%$ (38\%) and obtained from grafting of styrene solutions, respectively containing 0, 2 and 4\% DVB, the IEC recorded 2.05, 2.08 and 2.10, which agrees very well with the theoretical value (2.18), suggesting that the aromatic rings of the grafted polystyrene have achieved full degree of sulfonation irrespective of crosslinking. The absence of any effect of crosslinking is because IEC is a function of number of aromatic rings of polystyrene which provide sulfonation sites. Similar behavior was also reported for DVB crosslinked polystyrene sulfonic acid membranes prepared by radiation-induced grafting of styrene onto FEP, PFA, and poly(vinylidene fluoride),PVDF, films [23-25].

\subsubsection{Swelling Behavior}

The swelling behavior represented by the water uptake (WU\%) of PE-g-PSSA membranes having various DVB contents in correlation with the grafting yield (Y\%) is shown in Figure 5. The WU\% of all membranes was found to increase with the increase in Y\%. This is due to the increase in the hydrophilicity imparted to the membranes as a result of incorporation of more sulfonic acid groups, which facilitates the formation of the water rich ionic domains. However, the crosslinked membranes displayed lower WU compared to non-crosslinked one and the degree of swelling is a function of DVB content, i.e. the increase in DVB content lead to a decrease in the water uptake. This behavior can be attributed to the formation of crosslinked structure that reduces chain mobility and water sorption. The increase in the crosslinking density adds more restriction to chain mobility and water sorption. Since the increase in the water uptake in non-linear in all membranes, the existing trend indicates that other factors such as crystallinity and graft distribution are involved in affecting the swelling behavior. This behavior is similar to that of respective radiation grafted membranes based on FEP [26] and PTFE film [27]. 


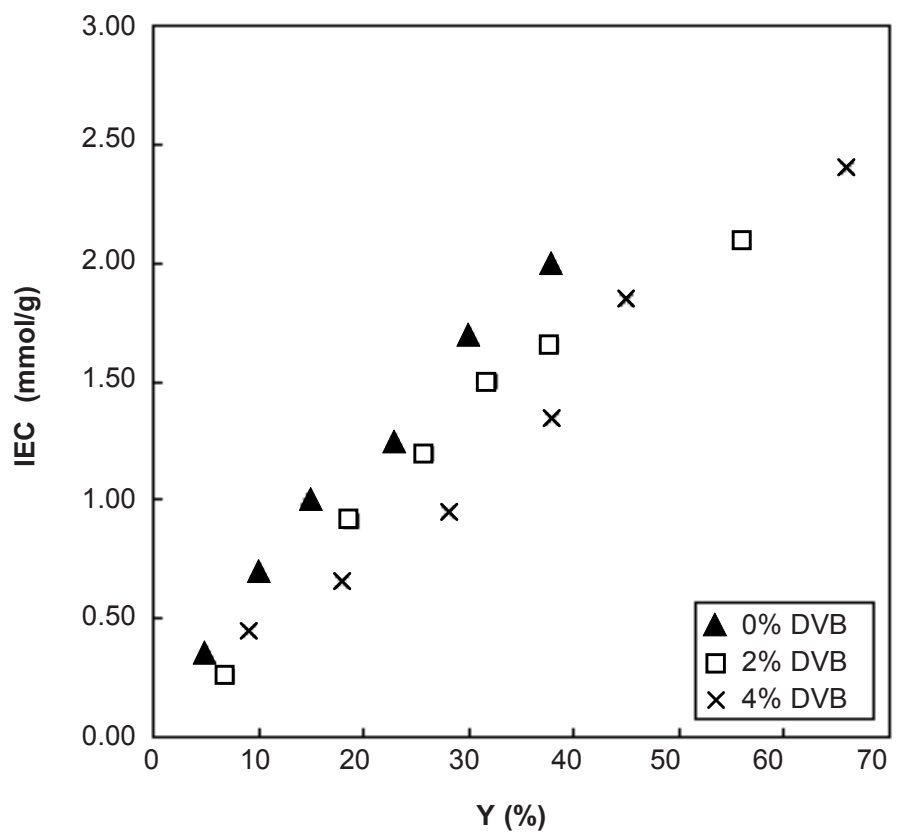

Figure 5 The relationship between swelling properties and grafting yield for PE-g-PSSA membranes having various DVB contents

\subsubsection{Ionic Conductivity}

Figure 6 shows the relationship between the ionic conductivity and the grafting yield (Y\%) in PE-g-PSSA membranes having various DVB contents at room temperature. Generally, the ionic conductivity in all membranes increases with $\mathrm{Y} \%$ and such increase is drastic at the beginning and tends to level off after achieving sufficient grafting levels $(28 \%)$ to attain an order of magnitude of $10^{-2} \mathrm{~S} / \mathrm{cm}$. Crosslinking of the membranes with 2 and 4\% DVB causes a remarkable decrease in the ion conductivity at all levels of grafting. The increase in the ionic conductivity with the increase in Y\% in all membranes is known to be due to the rise in both IEC and WU\%, leading to an increase in the number of ionic sites $\left(\mathrm{SO}_{3}^{-}\right)$and their swelling domains. This provides better environment for the ionic mobility in the membranes. Therefore, the high ionic conductivity demonstrated by these membranes at sufficient grafting (28\%) suggests that the water swollen ionic domains in the membranes tend to form network structure. Inversely, the lower value of the ionic conductivity at low $\mathrm{Y} \%$ is caused the diffusion limitation resulted from the segregation in the ionic domains, which remain bound to the membrane surfaces. As Y\% increases to sufficient level (28\%), the ionic domains become more inter-connected and reach the percolation threshold beyond which the diffusion limitations are overcome in a way that allows the ionic conductivity to reach a maximum value. In the mean time, the reduction in the conductivity caused by crosslinking can be ascribed to the reduction in the water swelling caused the restriction of PSSA chain mobilities by DVB crosslinks which eventually hiders ionic mobility in the membranes. 


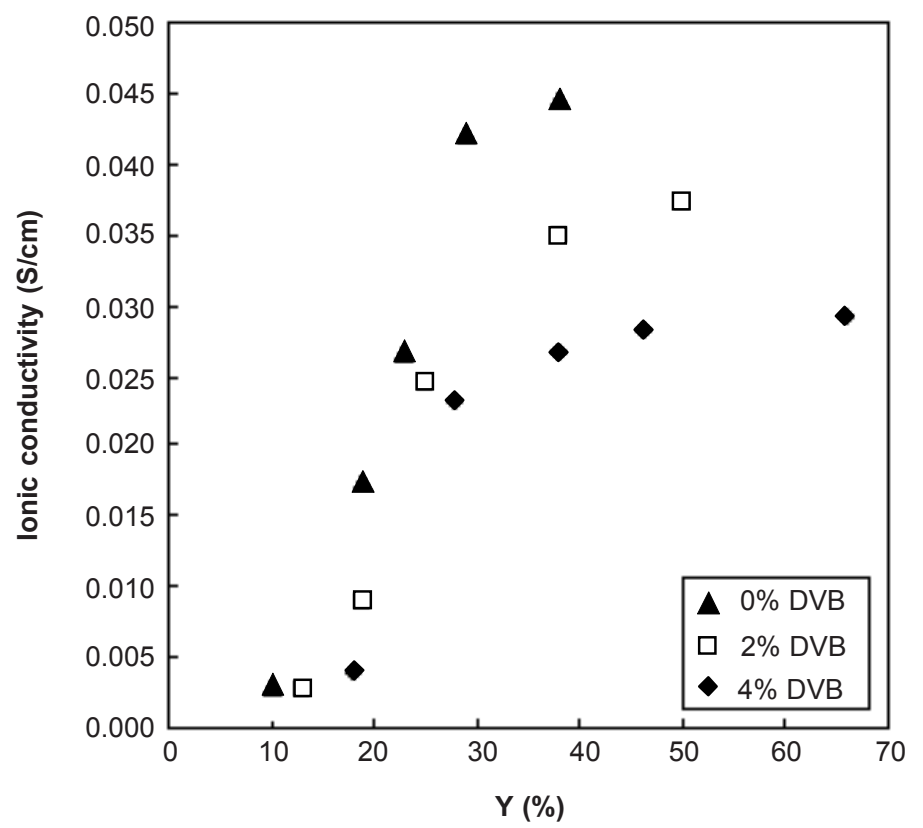

Figure 6 The relationship between ionic conductivity and grafting yield for PE-g-PSSA membranes having various DVB contents

These results are in a complete agreement with those reported by Momose et al. for similar membranes prepared by grafting trifluorostyrene onto low density PE films followed by sulfonation [13]. These membranes were found to achieve a homogenous grafting distribution and low resistance at $\mathrm{Y}=28 \%$ and above.

To summarize the effect of crosslinking on the physico-chemical properties of PE-g-PSSA membranes is represented by three samples having the same grafting yield (38\%) and prepared from grafting of styrene mixtures having 0, 2 and 4\% DVB under similar grafting conditions, as shown in Table 1. As can be seen, crosslinking is found to reduce the water uptake and the number of water molecules per sulfonic acid groups $\left(\mathrm{nH}_{2} \mathrm{O} / \mathrm{SO}_{3}{ }^{-}\right)$of the membranes and such effect depends on the crosslinking level. This is due to partial restriction imposed on polystyrene molecular chains by DVB crosslinks, which becomes more restrictive with the increase in crosslinking level (represented here by the increase in DVB in the bulk grafting solution from 2 to 4\%). On the other hand, the reduction in swelling caused by crosslinking was also found to decrease the ionic conductivity of the crosslinked membranes. Unlikely, ion exchange capacity, which depends on the amount of the incorporated polystyrene grafts was found to be independent on the level of crosslinking. This results indicate that crosslinking plays an important role in improving some essential properties of the membranes.

Table 1 Summary of physico-chemical properties of PE-g-PSSA membranes having various DVB contents. $\mathrm{Y}=38 \%$ in all membranes

\begin{tabular}{|c|c|c|c|c|}
\hline Membrane samples & W (\%) & $\mathrm{nH}_{2} \mathrm{O} / \mathrm{SO}_{3}^{-}$ & IEC (meq/g) & $\sigma(\mathrm{S} / \mathrm{cm}) \times 10^{-2}$ \\
\hline PE-g-PSSA (0\% DVB) & 40 & 11 & 2.10 & 3.7 \\
\hline PE-g-PSSA (2\% DVB) & 33 & 9 & 2.15 & 2.9 \\
\hline PE-g-PSSA (4\% DVB) & 27 & 7 & 2.05 & 2.7 \\
\hline
\end{tabular}


However, the level of crosslinking has to be optimized to maintain a balance between the desired (high ionic conductivity) and the undesired (excess swelling) properties in the membranes. The results of this work agrees very well with the findings of Gupta et al. [28] who studied crosslinking of FEP-g-PSSA membranes with DVB.

\subsubsection{Chemical Stability of the Crosslinked Membranes}

The effect of crosslinking on the chemical stability of PE-g-PSSA membranes having various DVB contents but the same grafting yield $(\mathrm{Y}=38 \%)$ is shown in Table 2. Crosslinking of the membranes with DVB was found to improve the chemical stability as indicated by the reduction in the loss in the weight and IEC of the membranes after being treated with $3 \% \mathrm{H}_{2} \mathrm{O}_{2}$ containing 4 ppm $\mathrm{Fe}^{2+}$ for 5 hours at $70^{\circ} \mathrm{C}$. This behavior can be reasonably attributed to the decrease in the swelling in the highly oxidative aqueous solution caused by crosslinking of polystyrene component in the membranes, leading to a reduction in the membrane chemical attack by $\mathrm{OH}$ radicals formed in the solution. In such membranes, the chemical attack by $\mathrm{OH}$ is reported to take place on the tertiary hydrogen of the $\alpha$-carbon of polystyrene side chain grafts with simultaneous loss of styrene (aromatic rings) and ionic species (sulfonic acid groups) i.e decomposition of the grafted polymer chains [29, 30]. Similar degradation trend was reported for polystyrene sulfonic acid resins [31]. Therefore, the chemical degradation is suggested to take place in the same manner despite of presence of PE component that is theoretically susceptible to chemical attach. If chemical took place on the PE component, one could not find the simultaneous reduction in weight loss and IEC of the treated. These results clearly show that crosslinking with DVB improves the stability of PE-g-PSSA.

Table 2 The effect of crosslinking on the chemical stability of PE-g-PSSA membranes having various DVB contents. $\mathrm{Y}=38 \%$ in all membranes

\begin{tabular}{lcc}
\hline Membrane samples & \multicolumn{2}{c}{ Membrane degradation } \\
& Weight loss (\%) & IEC (meq/g) loss \\
\hline PE-g-PSSA (0\% DVB) & 55 & 1.05 \\
PE-g-PSSA (2\% DVB) & 35 & 0.80 \\
PE-g-PSSA (4\% DVB) & 22 & 0.50 \\
\hline
\end{tabular}

\subsubsection{Structural and Thermal Properties of the Crosslinked Membranes}

Figure 7 shows the DSC thermograms of original PE film, PE- $g$-PS film, PE- $g$-PSSA and PE- $g$-PSSA/ DVB membranes. The two investigated membranes and the grafted film have the same grafting yield $(38 \%)$. The data obtained from these thermograms is summarized in Table 3. The melting temperature $\left(T_{\mathrm{m}}\right)$ of PE was found to be marginally reduced in PE-g-PSSA and PE-g-PSSA/DVB membranes unlike that of PE-g-PS films, which remained almost unvaried indicating that no significant changes (disorder) took place in the crystallites of $\mathrm{PE}$, since $T_{\mathrm{m}}$ is a function changes in the inherent crystallinity.

The enthalpy of melting $\left(\Delta H_{\mathrm{m}}\right)$ obtained from the area under the melting peaks was in the decreasing order of PE $>$ PE- $g$-PS $>$ PE- $g$-PSSA. This means grafting of styrene, styrene/DVB and the subsequent sulfonation caused a reduction in $\Delta H_{\mathrm{m}}$ of PE matrix. However, $\Delta H_{\mathrm{m}}$ of the DVB crosslinked membrane (PE-g-PSSA/DVB) is matching that of PE-g-PSSA membrane (as seen in Table 3) 


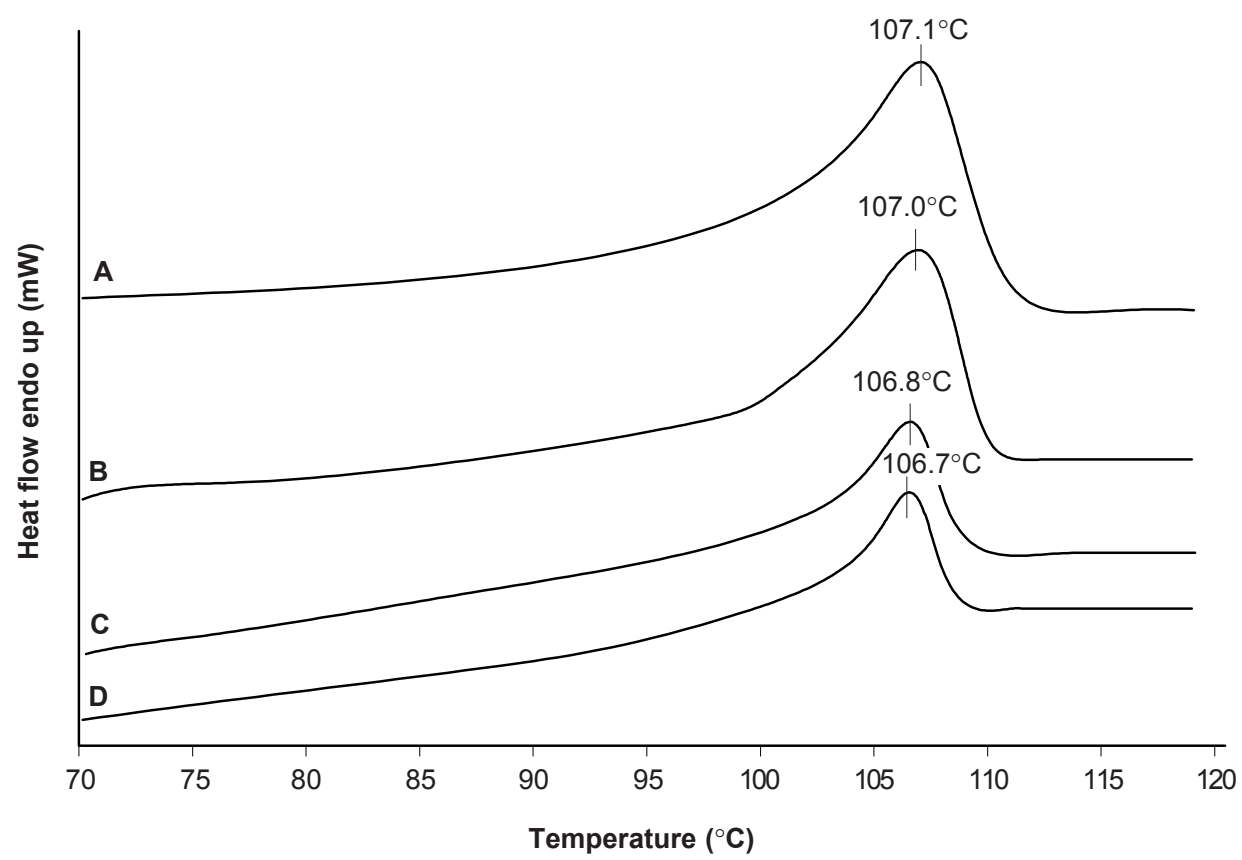

Figure 7 DSC thermograms of: A) original PE film, B) PE-g-PS film (Y=38\%), C) PE-g-PSSA membrane $(\mathrm{Y}=38 \%)$ and D) PE-g-PSSA/DVB membrane ( $\mathrm{Y}=38 \%)$

Table 3 Summary of DSC data of crosslinked and non-crosslinked PE-g-PSSA membranes. Original $\mathrm{PE}$ and PE-g-PS films were used as references

\begin{tabular}{lccc}
\hline Samples & $\boldsymbol{T}_{\boldsymbol{m}}$ & $\boldsymbol{H}_{\boldsymbol{m}}$ & $\boldsymbol{X}_{\boldsymbol{c}}$ \\
\hline PE & 107.1 & 63.1 & 21.8 \\
PE-g-PS* & 107.0 & 43.5 & 13.2 \\
PE- $g$-PSSA $^{*}(0 \%$ DVB) & 168.5 & 17.9 & 8.1 \\
PE-g-PSSA $^{*}(4 \%$ DVB) & 168.7 & 17.5 & 7.8 \\
\hline
\end{tabular}

$* \mathrm{Y}=38 \%$

suggesting that crosslinking of polystyrene (PS) with DVB during grafting reactions has no significant effect on the crystallities of PE film. This indicated that grafting and crosslinking take place in the amorphous region and at the surfaces of the crystallities. This finding was confirmed by the reduction in the degree of crystallinity $(\chi)$, which was found to be in the order of PE $>$ PE-g-PS $>$ PE-g-PSSA $>$ PE$g$-PSSA/DVB. The overall decreasing trend in $\Delta H_{\mathrm{m}}$ and $\chi$ caused by grafting and crosslinking can be understood based on the fact that grafting reaction was carried out at room temperature $\left(28^{\circ} \mathrm{C}\right)$, which is far below the $T_{\mathrm{m}}$ of low density PE film that has a very low swelling tendency hindering the diffusion of grafting solution (styrene and DVB) at room temperature according to Ishigaki et al. [32] and Gupta and Chapiro [33]. Consequently, it can be suggested that styrene/DVB diffuses only through the amorphous part of the PE matrix causing no swelling in the crystallites and forms crosslinked PS grafts in the entire amorphous region and around the surface of the crystallites despite the presence of the radicals in both crystalline and amorphous regions. This leads to a remarkable increase in the amorphous content, which exerts a dilution effect on the crystalline structure without 
any significant disruption in the inherent crystallites of PE matrix. It is also in accordance with the fact that grafting of styrene diluted with 1,2-dichloromethane in the present system is a diffusioncontrolled process whereby grafting proceeds by front mechanism [16]. On the other hand, Gupta and Scherer [26] found that the inherent crystallinity of fully fluorinated FEP in the membranes prepared using simultaneous irradiation and grafting with styrene did not show any change over the entire range of grafting levels (6.5-40\%). The study concluded that the grafts were introduced in the non-crystalline regions of the FEP matrix and therefore, they did not disrupt the inherent crystallites during the grafting reaction. On contrary, the overall crystallinity reduction in the sulfonated polystyrene grafted membranes based on partially fluorinated poly(vinylidene fluoride) (PVDF-gPSSA) membranes was found to be due to the decrease in the inherent crystallinity of PVDF and decrease in the crystallinity caused by dilution with amorphous polystyrene grafts [34]. This leads to the conclusion that the discrepancy in the response of the crystallinity to grafting of polystyrene towards hydrocarbon polymer (PE) to fluorocarbon one (PVDF) depending on the polymer matrix chain mobility difference during the grafting reaction.

\subsubsection{Thermal Stability of the Crosslinked Membranes}

Figure 8 shows TGA thermograms of original PE film, PE-g-PS film, PE-g-PSSA and PE-g-PSSA/ DVB membranes. The original PE and PE- $g$-PS films (thermograms a and $b$ ), undergo single step degradation pattern starts at $420^{\circ} \mathrm{C}$ in the former and $390^{\circ} \mathrm{C}$ in the latter due to main polymer chain decomposition. This indicates that the grafted PS is highly compatible with PE matrix in a way forming a single phase and therefore, it is difficult to discriminate the degradation temperature of $\mathrm{PE}$ from PS components in the grafted film. Nevertheless, grafting of PS was found to reduce the

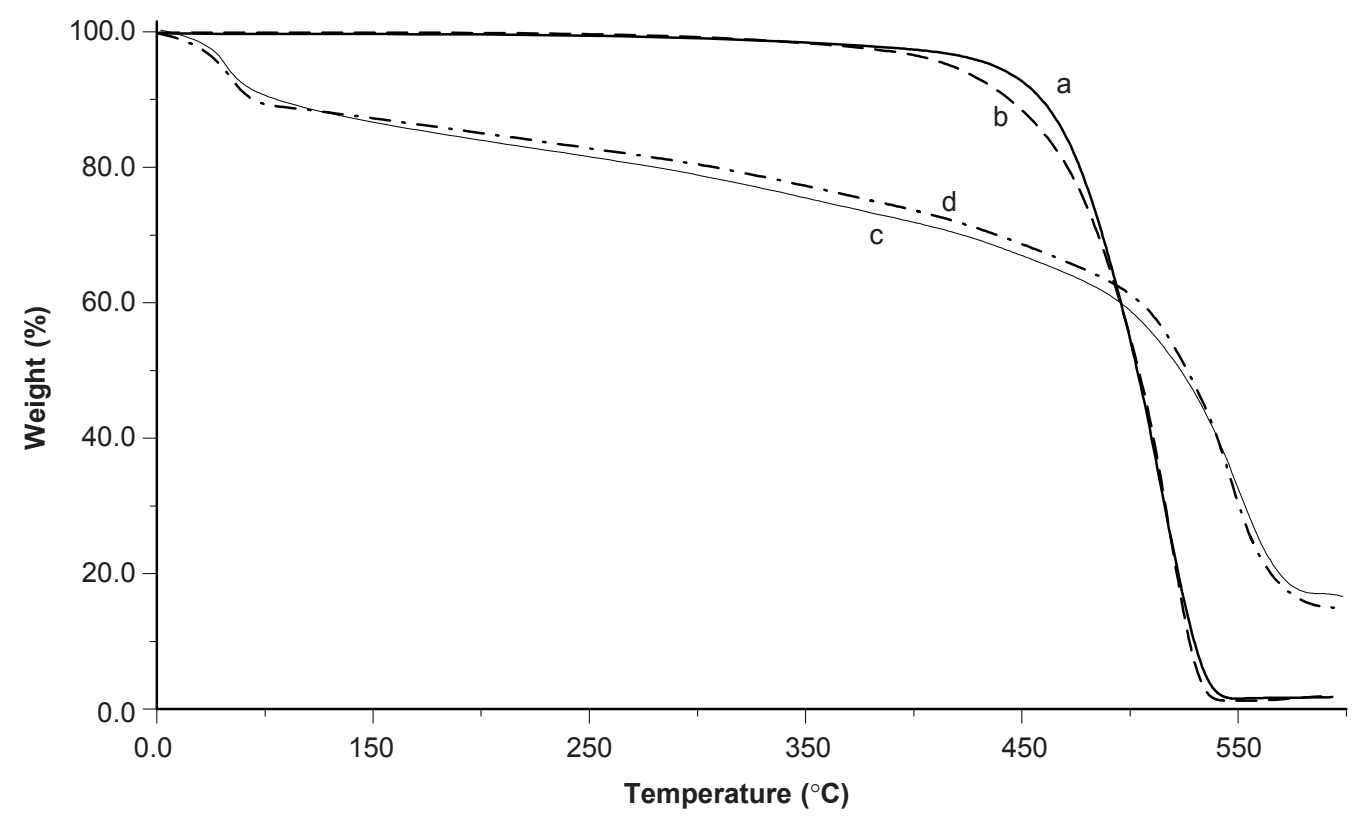

Figure 8 TGA thermograms of: a) original PE film, b) PE-g-PS film (Y=38\%), c) PE-g-PSSA membrane $(\mathrm{Y}=38 \%)$ and $\mathrm{d})$ PE-g-PSSA/DVB membrane $(\mathrm{Y}=38 \%)$ 
decomposition temperature by $30^{\circ} \mathrm{C}$, suggesting that grafting reduces the thermal stability of $\mathrm{PE}$ films.

On the other hand, the thermal stability behavior of PE-g-PSSA membrane and PE-g-PSSA/DVB membrane (thermograms $\mathrm{c}$ and $\mathrm{d}$ ) displayed a three-step degradation pattern represented by dehydration, desulfonation, and decomposition of main PE polymer chains. The removal of water (dehydration) starts at $50^{\circ} \mathrm{C}$ and continues up to $\sim 150^{\circ} \mathrm{C}$ under the influence of the hydrogen bonding exists between water molecules and sulfonic acid groups available in the membranes. The desulfonation of the membranes takes place at a temperature range of $160-300^{\circ} \mathrm{C}$. In the final stage, the polymer chains (comprising PE and PS or PE, PS and DVB) undergo decomposition. However, the decomposition temperature and the weight loss of the crosslinked PE-g-PS/DVB membrane were found to vary from the corresponding PE-g-PSSA (non-crosslinked) membrane. For instance, the decomposition temperature increased from $390^{\circ} \mathrm{C}$ in PE-g-PSSA to $410^{\circ} \mathrm{C}$ in PE-g-PS/DVB membrane. Moreover, crosslinking of the membrane was found to reduce the weight loss by around $5 \%$ indicating a slight improvement in the thermal stability of the PE- $g$-PS component of the membrane by crosslinking. The residues at the end of the thermogram were essentially found to be coke which increased by crosslinking. The overall results of thermal stability investigations of these membranes suggest that crosslinking with DVB has no direct effect on the thermal stability of the membranes represented by the decomposition of the functional groups, which withstand temperature up to 160 ${ }^{\circ} \mathrm{C}$. Nevertheless, this temperature permits the use of these membranes in various electrochemical applications. The multi-step decomposition behavior of the present membranes is in a complete agreement with that of cation exchange membranes based on partially fluorinated polymers such as PVDF [35] and fully fluorinated polymer such as PTFE [36], FEP [37] and PFA [38].

\subsection{CONCLUDING REMARKS}

Crosslinked cationic membranes (PE-g-PSSA/DVB) were successfully prepared by radiation-induced grafting of styrene/DVB mixture onto low density PE followed by sulfonation reaction. After investigating the effect of addition of crosslinking on the grafting behavior and the various properties of these membranes, the following concluding remarks can be drawn.

(i) The addition of DVB (2 and 4\%) to styrene monomer in presence of diluent was found to enhance the grafting yield.

(ii) The swelling and ionic conductivity of the membranes were reduced by the addition of DVB, and such reduction is a function of the crosslinking level.

(iii) The PE-g-PSSA/DVB membranes achieved an ionic conductivity in the order of magnitude of $10^{-2} \mathrm{~S} / \mathrm{cm}$ at sufficient grafting distribution ( $\mathrm{Y}=28 \%$ and above) and such magnitude is high enough to promote these membranes for various practical electrochemical applications.

(iv) Grafting and crosslinking of polystyrene were found to take place mainly in the amorphous region of the PE films, causing a dilution effect on the crystalline structure of the membranes.

(v) Crosslinking is also found to remarkably enhance the chemical stability of the membranes.

(vi) Crosslinking does not have any significant impact on the thermal stability represented by decomposition of sulfonic acid group despite causing some improvement in the thermal stability of PE-g-PS component of the membranes. 


\section{ACKNOWLEDGEMENTS}

The authors wish to acknowledge the financial support by the Malaysian Ministry of Science, Technology and Environment through IRPA program. Thanks are due to Dr. Khairul Zaman Mohd Dahalan and Kamaruddin Hashim from Malaysian Institute for Nuclear Technology Research (MINT) for their assistance during irradiation of samples.

\section{REFERENCES}

[1] Nasef, M. M. and E. A. Hegazy. 2004. Preparation and Applications of Ion Exchange Membranes by Radiation-induced Graft Copolymerization of Polar Monomers onto Non-polar Films. Prog. Polym. Sci. 29: 499-561.

[2] Oren, Y., V. Freger, and C. Linder. 2004. Highly Conductive Ordered Heterogeneous Ionexchange Membranes. J. Membr. Sci. 239: 17-26.

[3] Xu, T. 2005. Ion Exchange Membranes: State of Their Development and Perspective. J. Membr. Sci. 263: 1-29.

[4] Hwang, G. -J. and H. Ohya. 1998. Preparation of Anion Exchange Membrane Based on Block Copolymers. Part I: Amination of the Chloromethylated Copolymers. J. Membr. Sci. 140: 195-203.

[5] Zippi, E. M. and G. W. Kabalka. 1996. Synthesis, Characterization and Pyrolysis of Poly (Styrene/ divinylbenzene) Derivatives. Carbon. 34: 1539-1547.

[6] Hu, K. Y., T. W. Xu, W. H. Yang, and Y. X. Fu. 2004. Preparation of a Novel Heterogeneous Cation Permeable Membranes from Blends of Sulfonated Poly (phenylene sulfide) and Poly (ether sulfone). J. Appl. Polym. Sci. 91: 167-175.

[7] Rozek-Galina, W. and W. Trochimczuk. 1985. Polyethylene/poly(styrene-co-divinylbenzene) Sodium Sulphonate Membranes. J. Membr. Sci. 22: 47-59.

[8] Molau, G. 1981. Heterogeneous Ion-exchange Membranes. J. Membr. Sci. 8: 309-330.

[9] Choi, Y., M.-S. Kang, S.-H. Kim, J. Cho, and S.-H. Moon. 2003. Characterization of LDPE/ Polystyrene Cation Exchange Membranes Prepared by Monomer Sorption and UV Radiation Polymerization. J. Membr. Sci. 223: 201-215.

[10] Chen, W., R. Mesrobian, D. Ballantine, D. Metz, and A. Glines. 1957. Graft Copolymers Derived by Ionizing Radiation. J. Polym. Sci. 23: 903-913.

[11] Shkolink, S. and D. Behar. 1982. Radiation-induced Grafting of Sulfonates on Polyethylene. J. Appl. Polym. Sci. 27: 2189-2196.

[12] Horsfall, J. and K. Lovell K. 2002. Synthesis and Characterization of Sulfonic Acid-containing Ion Exchange Membranes Based on Hydrocarbon and Fluorocarbon Polymers. Eur. Polym. J. 38: 1671-1682.

[13] Momose, T., I. Ishigaki, and J. Okamoto. 1988. Radiation Grafting of $\alpha, \beta, \beta$-trifluoroethylenesulfonyl Fluoride onto Low-density Polyethylene Film by Simultaneous Irradiation Method. I. Kinetic Study of Simultaneous-irradiation Grafting. J. Appl. Polym. Sci. 36: 55-62.

[14] Momose, T., K. Tomiie, I. Ishigaki, and J. Okamoto. 1989. Radiation Grafting of $\alpha, \beta$, $\beta$-trifluorostyrene onto Various Polymer Films by Preirradiation Method. J. Appl. Polym. Sci. 37: 2165-2168.

[15] Tsuneda, S., K. Saito, T. Sugo, and K. Makuuchi. 1995. Protein Adsorption Characteristics of Porous and Tentacle Anion-exchange Membrane Prepared by Radiation-induced Graft Polymerisation. Radiat. Phys. Chem. 46: 239-245. 
[16] Nasef, M. M., H. Saidi, H. M. Nor, K. M. Dahlan, and K. Hashim. 1999. Radiation-induced Grafting of Styrene onto LDPE for Preparation of Cation Exchange Membranes. I. Effect of Grafting Conditions. J. Nucl. Sci. (Malaysia). 1: 55-63.

[17] Nasef, M. M., H. Saidi, H. M. Nor, K. M. Dahlan, and K. Hashim. 2000. Radiation-induced Grafting of Styrene onto LDPE for Preparation of Cation Exchange Membranes. II. Properties of the Sulfonated Membranes. J. Nucl. Sci. (Malaysia). 18: 47-57.

[18] Zu, J., M. Wu, H. Fu, and S. Yao. 2005. Cation-exchange Membranes by Radiation-induced graft Copolymerization of Monomers onto HDPE. Radiat. Phys. Chem. 72: 759-764.

[19] Reddy, P. R. S., G. Agathian, and A. Kumar. Preparation of Strong Acid Cation-exchange Membrane Using Radiation-induced Graft Polymerization. Radiat. Phys. Chem. 73: 169-174.

[20] Nasef, M. M. and H. Saidi. 2004. Structure of Polyethylene-graft-polystyrene Sulfonic Acid Membranes Prepared by Radiation-induced Grafting. Int. J. Polym. Mater. 53: 1-17.

[21] Richardson, M. J. 1972. Precision Differential Calorimetry and the Heat of Fusion of Polyethylene. J. Polym. Sci., Part C. 251-269.

[22] Elmidaoui, A., A. Cherif, J. Brunea, F. Duclert, T. Cohen, and C. Gavach. 1992. Preparation of Perfluorinated Ion-exchange Membranes and Their Application in Acid Recovery. J Membr. Sci. 67: 263-271.

[23] Gupta, B., F. Büchi, M. Staub, D. Grman, and G. G. Scherer. 1996. Cation Exchange Membranes by Pre-irradiation Grafting of Styrene into FEP Films. II. Properties of Copolymer Membranes. J. Polym. Sci.: Pt A: Polym. Chem. 34: 1873-1880.

[24] Nasef, M. M. and H. Saidi. 2003. Preparation of Crosslinked Cation Exchange Membranes by Radiation Grafting of Styrene/divinylbenzene Mixtures onto PFA Films. J. Membr. Sci. 216: 2738.

[25] Holmberg, S., T. Lehtinen, J. Naesman, D. Ostrovskii, M. Paronen, R. Serimaa, F. Sundholm, G. Sundholm, L. Torell, and M. Torkkeli. 1996. Structure and Properties of Sulfonated Poly[(vinylidene fluoride)-g-styrene] Porous Membranes. J. Mater. Chem. 6: 1309-1317.

[26] Gupta, B., F. N. Büchi, and G. G. Scherer. 1994. Development of Radiation FEP-g-polystyrene Membrane: Some Property-structure Correlations. Polym. Adv. Technol. 5: 493-498.

[27] Nasef, M. M. 2002. Structural Investigation of Radiation Grafted PTFE-g-polystyrene Sulfonic Acid Membranes. Euro. Polym. J. 38: 87-95.

[28] Gupta, B., F. Büchi, G. Scherer, and A. Chapiro. 1996. Crosslinked Ion Exchange Membranes by Radiation Grafting of Styrene/Divinylbenzene into FEP Films. J. Membr. Sci. 118: 231-238.

[29] Assink, R., J. C. Arnold, and R. Hollandsworth. 1991. Preparation of Oxidatively Stable Cationexchange Membranes by the Elimination of Tertiary Hydrogens. J. Membr. Sci. 56: 143-151.

[30] Büchi, F. N., G. Gupta, O. Haas, and G. G. Scherer. 1995. Study of Radiation-grafted FEP-gpolystyrene Membranes as Polymer Electrolytes in Fuel Cells. Electrochimica Acta. 40: 245353.

[31] Hodgdon, R., J. R. Boyack, and A. B. La Conti. 1966. Advance Development and Laboratory Technical Report No. 65DE5. General Electric Co. West Lynn., MA, USA.

[32] Ishigaki, I., T. Sugo, K. Senoo, and T. Okada. 1982. Graft Polymerization of Acrylic Acid onto Polyethylene Film by Preirradiation Method. I. Effects of Preirrradation Dose, Monomer Concentration, Reaction Temperature, and Film Thickness. J. Appl. Polym. Sci. 27: 1033-1041.

[33] Gupta, B. and A. Chapiro. 1989. Preparation of Ion-exchange Membranes by Grafting Acrylic Acid onto Pre-irradiated Polymer Films-1. Grafting into Polyethylene. Eur. Polym. J. 25: 11371143.

[34] Hietala, S., S. Holmberg, M. Karjalainen, J. Näsman, M. Paronen, R. Serimaa, F. Sundholm, and S. Vahvaselka. 1997. Structural Investigation of Radiation Grafted and Sulfonated Poly(vinylidene fluoride) PVDF Membranes. J. Mater. Chem. 5: 721-727. 
[35] Hietala, S., M. Keol, M. Elomaa, and F. Sundholm. 1998. Thermal Stability of Styrene Grafted and Sulfonated Proton Conducting Membranes Based on Poly(vinylidene fluoride). J. Matter. Chem. 8: 1127-1132.

[36] Nasef, M. M. 2000. Thermal Stability of Radiation Grafted PTFE-g-polystyrene Sulfonic Acid Membranes. Polym. Degrad. Stab. 68: 231-238.

[37] Gupta, B. and G. G. Scherer. 1993. Proton Exchange Membranes Prepared by Radiation Grafting of Styrene onto FEP Films. I. Thermal Characteristics of Copolymer Membranes, G. J. Appl. Polym. Sci. 50: 2129-2134.

[38] Nasef, M. M., H. Saidi, and H. M. Nor. 2000. Cation Exchange Membranes by Radiationinduced Graft Copolymerization of Styrene onto PFA Copolymer Films. III. Thermal Stability of the Membranes. J. Appl. Polym. Sci. 77: 1877-1885. 